\title{
'I AM NOT A MONSTER': THE LINGUISTIC STIGMA OF BORDERLINE PERSONALITY DISORDER
}

\author{
Kaja Widuch \\ University College London (UK)
}

\begin{abstract}
Borderline Personality Disorder is arguably the most distressing disorder amongst the DSM diagnoses for all involved. Although psychiatric labelling can be validating it is often stigmatising. Due to the nature of BPD, people living with the disorder (PBPD) tend to be marginalized and discriminated against. A quick and random review of the World Wide Web (including a selection of popular social media platforms) reveals a common linguistic theme in describing BPD. PBPD are 'toxic', 'difficult' and 'manipulative. Other labels, more diagnostically - oriented see PBPD as the 'PDs' or 'the borderlines'. These also carry negative connotations of the inner and outer groups - 'us' vs 'them'. Given the nature of the labels, recovery for PBPD is often dubious. One might think - 'I am a monster anyway', a classic example of cognitive dissonance. The language used in clinical practice as well as out of it is a powerful weapon. Some might poetically describe BPD as a lethal cocktail of blended psychopathologies with the ingredients including chronic suicidality, abandonment and intermittent lucidity to name a few. Of note, externalising such pathologies in an adaptive way is almost a fantasy for the therapy team. A more user friendly descriptive diagnosis is 'difficulty in emotion regulation'. However, probably the most accurate 'label' of BPD for PBPD is 'living in acute pain'. The current climate and the uncertainty surrounded the ongoing COVID-19 pandemic has meant a significantly increased risk not only in symptoms remission but also in the increase in cyber-bullying and suicidality rate. The pandemic has also put a halt to the Participant and Public Involvement in the evidence based practice. Linguistic shift in reducing stigma is essential and of immediate need.
\end{abstract}

Keywords: Borderline personality disorder, BPD, stigma, linguistic-labelling, linguistic-shift.

\section{Introduction}

Borderline Personality Disorder (BPD) is a disorder of chronic instability, impulsivity and hypersensitivity to possible rejection and abandonment (American Psychiatric Association, 2013). A key to understanding BPD is recognizing its 'socio-emotional' vulnerability is organic, with most symptoms triggered, exacerbated and perpetuated by intimate relationships. Evidence to date attributes the aetiology of BPD to early developmental traumas, experienced pathologies and caregiver neglect (e.g. Zanarini et al., 1997). The inherent emotional dysregulation means PBPD are particularly vulnerable to harm from self and others through maladaptive coping behaviors or stigma (e.g. suicidal attempts, 'trolling').

Historically, BPD has been highly stigmatized, predominantly due to its complex nature and poor patient outcomes (Aviram et al., 2006). Linguistically, BPD stigma has formed through language evolution i.e, when a denotative meaning adopted a new negative connotative meaning (e.g. evil) The 'border line' label itself was first originated by Adolph Stern (1938) a pioneer psychoanalyst who described PBPD as 'extremely difficult to handle'. Ever since, BPD stigma advanced beyond 'difficult'. The Diagnostic and Statistical Manual of Mental Disorders (DSM-5; APA, 2013) for instance openly refers to individuals with BPD as 'manipulative'. Stigmatising labels of BPD can lead to 'spoilt identity' (Goffman, 1963) or self-inflicted via cognitive dissonance (Festinger,1962).

Functionally, stigma itself has a segregative nature (Tajfel \& Turner, 1986). Literature suggests there is a particular 'splitting' jargon used to speak of PBPD. Although little is known of linguistic-stigma of BPD, Interdisciplinary literature on negativity bias (Rozin \& Royzman, 2001) effects of hurtful words (Vangelisti \& Young 2000) and toxic speech (Tirrell, 2018) suggest language is a powerful and destructive tool. Crocker \& Smith (2019) previously pointed out the existing need of linguistic-shift in current mental health practice and disabilities care. 
As linguistic-stigma is a novel concept this project aimed to explore the semantic profile associated with BPD stigma. Data gathered was interpreted using qualitative methodology from linguistic standpoint and discussed in terms of BPD vulnerability and existing cross-disciplinary knowledge.

\section{Methods}

\subsection{Literature review}

To establish a stereotypical profile of a person living with BPD, peer-reviewed publications were reviewed for content. The review was initiated with a keyword search using Boolean operators followed by a backward and forward citation search. The desired key terms were 'borderline personality disorder' AND 'stigma' OR 'stereotype'.

\subsection{Autocomplete engine search}

Three top search engines were used to examine trends. (Google, Bing and Yahoo). Search suggestions were tested for the following search string variations: 'borderline personality disorder is', 'borderline(s) is/are' and 'BPD(s) is/are'. To avoid bias, the researcher's browsing history was cleared prior to each search and all search activities were carried out in the Incognito mode.

\subsection{Social media review}

Tweets, posts and comments were scrutinized for stigmatizing content across the three popular social media platforms (Twitter, Facebook and Instagram) targeting 'Borderline Personality Disorder', 'BPD(s)' and 'borderline(s)' keywords and hashtags. Snowballing method was applied to the primary search results.

\section{Results}

The results suggest the linguistic-stigma of BPD is multidimensional:

1. Dehumanizing via diagnostic labelling

2. Out-casting via antagonistic lexemes (and diagnostic labelling)

3. Entrenched via semantic shift

4. Intensified via figurative speech

5. Via BPD analogy

Figure 1. Linguistic profile of a person living with Borderline Personality Disorder.

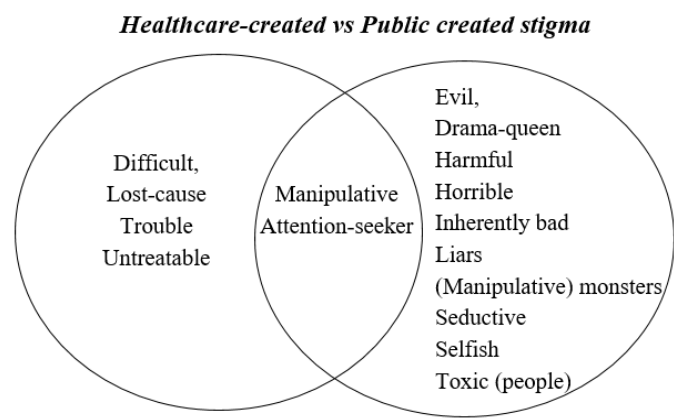

\section{Discussions and conclusions}

The results of this pilot project provide preliminary evidence of a distinctive, multidimensional linguistic profile of BPD stigma. Although plausible, it should be noted the results are presumptive and a mere snapshot of current lexicalization patterns. It should also be acknowledged, it was not possible to extrapolate demographic characteristics of the data collected. Age, educational level and lived experience of BPD may be important predictors of semantic-shift in stigmatisation process.

There are evident disparities in the linguistic focus of BPD across therapeutic and public settings. While diagnostic labels lead the psychiatric medical model, psychological therapies prefer phenomenologically oriented descriptive case-conceptualization approach. Functionally, diagnostic labelling does aid evidence-base-practice and thus the choice of therapeutic intervention. BPD 'membership' on the other hand allows the coalition of support groups and networking among PBPD. Yet, these labels are still a powerful tool used to segregate psychiatric from non-psychiatric populations 
(Goffman, 1963 Paradoxically, there is an analogy between the BPD and labelling - labels are 'splitting' (they either good or bad).

The cluster of lexemes used to describe PBPD available in the public domain appears to be more antagonistic and critical compared to statements reported by healthcare professionals reported through research publications. Intersecting labels across the two domains seems to be minimal and these tend to be stereotypical (e.g. attention-seeker, manipulative). The public tend to be rather liberal in BPD lexicalisation and depict PBPD as malevolent individuals while healthcare professionals see these clients as inconvenience, a hopeless case or a nuisance. However, it cannot be concluded the derogatory statements, available in the virtual public domain do not belong to healthcare professionals (e.g. tweets).

The diversity of lexical variation in English language enabled linguistic stigma of BPD to evolve and ingrain further through sematic shift - the adjective 'toxic' is now commonly associated with BPD or BPD relationships. Moreover, BPD lexicalisation seems to be intensified via sophisticated epithets (toxic people) metaphors (evil, monsters) or alliterations (manipulative monster). Though, it was not the primary focus of this project, it was noted during data collection the stigmatizing lexemes evoke feelings of rejection, anger, hurt and frustration among PBDP. Such reaction to hate speech is congruent with Vangelisti \& Young (2000) notion 'words hurt' and 'us/them dichotomization' (Tajfel \& Turner 1986; Tirrell, 2018). The power of stigma seems to have also resulted 'spoilt identities' (Goffman, 1963) with many PBPD identifying as 'toxic'. Others might defend and excuse their BPD labels through cognitive dissonance mechanism (Festinger,1962) - 'I am incurable anyway so therapy is pointless'.

Endorsing the antagonistic language towards people living with BPD appears to contribute to maintaining mental health stigma, social isolation and barrier to therapy. Eliminating social stigma should be shared responsibility among service users, public and professionals alike. Given the hypersensitive nature of BPD public health policy makers should consider implementing stern boundaries across social media platforms, search engines and professional resources to protect this particularly vulnerable group who are at significant risk to own's safety, especially now during current pandemic climate. Universal linguistic-shift of compassion, empathy, mindfulness and respect would be a significant step in eradicating the use of dehumanizing and prejudicial statements towards PBPD.

The follow up study will seek to develop a standardized reliable instrument to measure the psychological effects of linguistic-stigma in people living with Borderline Personality Disorder.

\section{References}

American Psychiatric Association (2013) Diagnostic and Statistical Manual of Mental Disorders: Diagnostic and Statistical Manual of Mental Disorders, Fifth Edition. Arlington, VA: American Psychiatric Association

Aviram RB, Brodsky BS, Stanley B. (2006) Borderline personality disorder, stigma, and treatment implications. Harvard Review of Psychiatry, 14(5)249-21.

Crocker, A.F., Smith, S.N. (2019). Person-first language: are we practicing what we preach? Journal of Multidisciplinary Healthchcare, 12: 125-129.

Festinger, L. (1962). Cognitive dissonance. Scientific American, 207(4): 93-107.

Goffman E. (1963) Stigma: Notes on the management of spoiled identity. Prentice-Hall; Englewood Cliffs

Rozin, P., Royzman, Edward B. (2001). Negativity bias, negativity dominance, and contagion. Personality and Social Psychology Review, 5 (4): 296-320.

Stern A. (1938) Psychoanalytic investigation of and therapy in the borderline group of neuroses. Psychoanalysis Quarterly, 7:467-489.

Tajfel, H. \& Turner, J. C. (1986). The social identity theory of inter-group behavior. In S. Worchel \& W. G. Austin (Eds.), Psychology of Intergroup Relations. Chicago: Nelson-Hall.

Tirrell, L., (2018) Toxic speech: Inoculations and antidotes. The Southern Journal of Philosophy 56, 116-144, 2018. 9,

Vangelisti, A. L., \& Young, S. L. (2000). When words hurt: The effects of perceived intentionality on interpersonal relationships. Journal of Social and Personal Relationships, 17(3), 393-424.

Zanarini, M. C., et al. (1997). Reported pathological childhood experiences associated with the development of borderline personality disorder. The American Journal of Psychiatry, 154(8), 1101-1106. 